\title{
The Legacy of Kurt Mahler
}

\section{Jonathan M. Borwein, Yann Bugeaud, and Michael Coons}

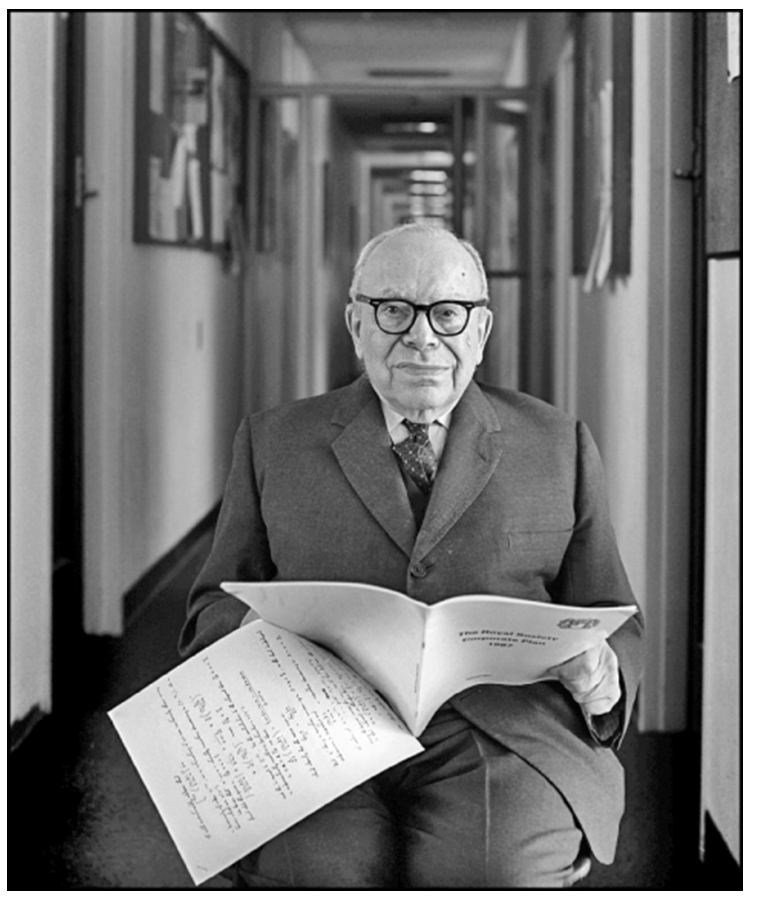

Kurt Mahler (1903-1988).

I hope that you can continue with your preparing of my collected works. When my old papers first appeared, they produced little interest in the mathematical world, and it was only in recent times that they have been rediscovered and found useful. So a collection of all my papers may repair this position!

- (Kurt Mahler, in his last letter to Alf van der Poorten)

The quote at the beginning of this article was taken from a letter written by Kurt Mahler to Alf van der Poorten the day before Mahler died on

Jonathan M. Borwein is a Laureate Professor of mathematics at the University of Newcastle. His email address is jonathan. borwein@newcast 1e.edu.au.

Yann Bugeaud is professor of mathematics at the Université de Strasbourg. His email address is bugeaud@math. unistra.fr.

Michael Coons is a senior lecturer and ARC research fellow at the University of Newcastle. His email address is michae1.coons@newcast 1e.edu.au.

DOI: http://dx.doi.org/10.1090/noti1241
February 25, 1988; it was received after van der Poorten had heard about Mahler's death.

At that time van der Poorten had already begun to collect Mahler's works. Over twenty-five years have now passed since Mahler's death, and nearly five years since van der Poorten's. Recently, in memory of van der Poorten, we ${ }^{1}$ finished van der Poorten's project and established the Kurt Mahler Archive. The Archive is hosted by the Centre for Computer-Assisted Research Mathematics and its Applications (CARMA), which is located ${ }^{2}$ at the University of Newcastle, Australia. It can be found using the following URL:

carma.newcastle.edu.au/mahler/index.htm7

\section{Mahler, the Man}

Mahler was born in Krefeld, Germany, July 20, 1903. He did not come out of an academic family but, nonetheless, from one which loved the printed word; his father ran a printing firm. While first entering academia in a nontraditional way, Mahler should most appropriately be considered a student of Siegel, and this is how the Mathematics Genealogy Project has recorded it. With the change of power in 1933, Mahler realized that he would need to leave Germany. After six weeks in Amsterdam, Mahler spent the academic year 1933-34 in Manchester under a fellowship secured by Mordell. He spent most of the next few years in the Netherlands until returning to the University of Manchester in 1937, where he remained for twenty-five years. After that, with the exception of a few years' hiatus in the United States of America, he was an Australian mathematician. ${ }^{3}$

\footnotetext{
${ }^{1}$ Joy van der Poorten helped immensely with collecting Mahler's harder-to-get papers as well as with the correspondence between Alf van der Poorten and Mahler. We thank her greatly for her contribution to the Archive.

${ }^{2}$ We are currently putting together a Mahler selecta with the help of Michael Baake (University of Bielefeld, Germany) and Reinout Quispel (La Trobe University, Australia).

${ }^{3}$ There are numerous biographies of Kurt Mahler, which are extremely well written. Thus we will not dwell too much on this here. See [2], [3], [5], [7], [17], [19], [22].
} 


\section{Mahler and Number Theory in Australia}

Under the invitation of B. H. Neumann, in 1962, Mahler visited Australia and, in particular, the then quite new Australian National University (ANU). He was impressed enough to quickly join the ANU as a professor in its Institute of Advanced Studies. As Mahler recollects:

There was at this time no teaching of number theory in the undergraduate school (School of General Studies) at the ANU. I therefore gave a course on this subject to second and third year students at the SGS, probably the first one ever in Canberra. One of my undergraduates, Coates, asked me to introduce him to research. I provided him with problems to work on, and by the time he obtained his BSc, he had already several papers published or in print.

-(Kurt Mahler, as quoted from [19])

One gains much more appreciation for Mahler reading his students' view of the same time.

Mahler was also very concerned with sowing the seeds of his own mathematical knowledge in his new country. As in his own mathematical research, he instinctively felt that the best way to do this was to go back to first principles, and to begin by teaching beginners in the subject. The ANU had begun to award undergraduate degrees only a few years before Mahler arrived, and Hanna Neumann was appointed to head the new Department of Mathematics in the teaching side of the University (the School of General Studies) at about the same time that Mahler took up his chair. Between them, they arranged for Mahler to give two courses to the small number of undergraduates reading mathematics, one in 1963 on elementary number theory, and the second in 1964 on the elliptic modular function $j(z)$. One of us had the good fortune to attend these courses. Mahler started and finished each lecture with extraordinary punctuality; in between, the audience was given a rare insight into his understanding of and enthusiasm for the material of the lecture. As he spoke, he would produce a beautiful written exposition on the blackboard of the key points, which were neatly placed in order in his characteristic rectangular boxes. Although he seemed at first so different and forbidding, we soon discovered that he was very willing to talk about his knowledge of mathematics in general, and to lend us his own mathematical books when we could not find them in the library. Mahler gave lectures at various summer schools in Canberra and elsewhere around Australia, as well as a number of advanced courses on transcendental number theory in the Institute of Advanced Studies. In the end the fascination of what he was doing beguiled us both into research in number theory, and we made our first steps in mathematical research on problems suggested by him.

-(John Coates and Alf van der Poorten, as quoted from [19])

These two students are well known to those of us in number theory, and many of us beyond. John Coates went on to become a Fellow of the Royal Society and was Sadleirian Professor of Pure Mathematics at Cambridge from 1986 to 2012. Soon after Mahler moved to Australia, at the encouragement of George Szekeres, Alf van der Poorten visited Mahler regularly in Canberra. A friendship based at first on a common interest in science fiction ${ }^{4}$ turned quickly into a mathematical relationship. Van der Poorten became Mahler's doctoral student, graduating ${ }^{5}$ in 1968 . Alf van der Poorten went on to positions at the University of New South Wales and then Macquarie University, both in Sydney.

Van der Poorten ${ }^{6}$ continued Mahler's legacy of number theory in Australia, both mathematically in a very interesting and important collaboration with John Loxton, and as a mentor and supporter of young Australian mathematicians ${ }^{7}$. One such instance remembered by a former young Australian mathematician goes as follows.

I first encountered Alf's unique style when I was a member of the Australian International Olympiad (IMO) team and we came across his wonderfully entertaining paper on Apéry's proof of the irrationality of $\zeta$ (3). I assumed from his name that he was Dutch, and was then pleasantly surprised when I met Alf at the IMO team send-off reception and

\footnotetext{
${ }^{4}$ This is entirely evident from the correspondence between Mahler and van der Poorten. The amount of space devoted to science fiction is roughly asymptotic to that devoted to mathematics.

${ }^{5}$ Alf van der Poorten was enrolled as a student at the University of New South Wales, not at the ANU.

${ }^{6}$ There is a comprehensive biography of Mahler's life and work written by David Hunt [6] in Number Theory and Related Fields. In memory of Alf van der Poorten. Based on the proceedings of the international number theory conference, Newcastle, Australia, March 12-16, 2012 [1].

${ }^{7}$ In keeping with van der Poorten's avid support of young Australian mathematicians, in his memory his family funds the Alf van der Poorten Travelling Fellowships, which aim to assist young pure mathematicians (who have earned a $\mathrm{PhD}$ in Australia) in travelling in Australia and overseas so that they can enrich their mathematical research through contact with other mathematicians.
} 
to find that he lived and worked in Sydney! He then and there told me what the $p$-adic numbers were and immediately offered me a job at any time in the future! I took him up on his offer at the end of my first year at Melbourne University, and spent six weeks in his office annex learning about elliptic curves, the Riemann-Roch theorem, the Weil conjectures, and, of course a lot of great stuff about recurrence relations (Skolem-Mahler-Lech) and continued fractions. I have always appreciated the time he spent talking to me-it was clear he cared a great deal about young Australian mathematicians.

-(Frank Calegari, as quoted from [6])

Mahler's contribution to number theory in Australia continues today, beyond his work and the work of his students and colleagues, in the Mahler Lectureship of the Australian Mathematical Society. Every two years, the Australian Mathematical Society honors Mahler's legacy by awarding a distinguished lectureship in his name.

The Mahler Lectureship is awarded every two years to a distinguished mathematician who preferably works in an area of mathematics associated with the work of Professor Mahler. It is usually expected that the Lecturer will speak at one of the main Society Conferences and visit as many universities as can be reasonably managed.

- (Australian Mathematical Society website: www. austms.org.au/p

The phrase visit as many universities as can be reasonably managed is not taken lightly. The 2013 Mahler Lecturer was Akshay Venkatesh of Stanford University. Professor Venkatesh gave sixteen talks in nineteen days at ten different universities throughout Australia. Giving so many talks in that amount of time is a difficult task. Include the 10,000+ kilometers of travel within Australia in addition to the 24,000 kilometers just to get to and from Australia from Stanford, and the Mahler Lectureship starts to sound like a daunting thing to accept (Akshay, if you are reading this, we all thank you heartily). Of course, Venkatesh grew up in Perth, so presumably he knew what he was getting into.

The 2015 Mahler Lecturer is Manjul Bhargava, recent Fields Medalist and professor of mathematics at Princeton University.

Former Mahler Lecturers comprise a marvelous group: John Coates (1991), Don Zagier (1993), Michel Mendes France (1995), Peter Hilton (1997), John H. Conway (1999), Robin Thomas (2001), Hendrik Lenstra (2003), Bruce Berndt (2005), Mark
Kisin (2007), Terence Tao (2009), ${ }^{8}$ and Peter Sarnak (2011).

\section{The Mahler Archive}

The way most of us interact with Mahler's legacy is now through his work. With the advent of the digital age, looking up papers is a much easier task than in previous times. With this in mind, we have made the Kurt Mahler Archive freely available online (see the first part of this article for the details and URL). It contains (in PDF format) every mathematical article published by Mahler, as well as a host of links to biographies and other information. His books are listed but are not available through the Archive.

Mathematically, it is quite easy to point out various highlights from Mahler's work. We do so here, hitting only a few of what we think are exceptional. Of course, this view is biased by our interests. For a more thorough list of topics see Mahler's "Fifty years as a mathematician" [16] or the obituaries by Cassels [3] or van der Poorten [22].

1. In [8] Mahler introduced a measure of the quality of approximation of a complex transcendental number $\xi$ by algebraic numbers. For any integer $n \geq 1$, we denote by $w_{n}(\xi)$ the supremum of the real numbers $w$ for which

$$
0<|P(\xi)|<H(P)^{-w}
$$

has infinitely many solutions in integer polynomials $P(x)$ of degree at most $n$. Here $H(P)$ stands for the naïve height of the polynomial $P(x)$, that is, the maximum of the absolute values of its coefficients. Further, we set

$$
w(\xi)=\limsup _{n \rightarrow \infty} \frac{w_{n}(\xi)}{n} .
$$

According to Mahler, we say that $\xi$ is

- an $S$-number if $w(\xi)<\infty$,

- a $T$-number if $w(\xi)=\infty$ and $w_{n}(\xi)<\infty$ for any integer $n \geq 1$,

- a $U$-number if $w(\xi)=\infty$ and $w_{n}(\xi)=\infty$ for some integer $n \geq 1$.

The terminology $S$-number may have been chosen to honor Siegel. Almost all numbers, in the sense of Lebesgue measure, are $S$-numbers, and Liouville numbers are examples of $U$-numbers. The existence of $T$-numbers remained an open problem for nearly forty years until it was confirmed by Schmidt [20], [21]. An important point

\footnotetext{
${ }^{8}$ In 2009 the lectureship was organized in partnership with the Clay Mathematics Institute, so the lecture tour that year was known as the Clay-Mahler lectures.
} 
in Mahler's classification is that two algebraically dependent transcendental numbers always fall in the same class.

2. One of the first significant contributions of Mahler is an approach, now called "Mahler's method," yielding transcendence and algebraic independence results for the values at algebraic points of a large family of power series satisfying functional equations of a certain type. In the seminal paper [18] Mahler established that the Fredholm series $f(z)=\sum_{k \geq 0} z^{2^{k}}$, which satisfies $f\left(z^{2}\right)=f(z)-z$, takes transcendental values at any nonzero algebraic point in the open unit disc.

3. Concerning specific transcendence results, in [10] Mahler proved that Champernowne's number

$$
0.12345678910111213141516 \cdots
$$

is transcendental and is not a Liouville number. Champernowne had proven it normal to base ten a few years earlier.

4. Mahler [13] was also the first person to give an explicit irrationality measure for $\pi$. He showed that, if $p$ and $q \geq 2$ are positive integers, then

$$
\left|\pi-\frac{p}{q}\right|>\frac{1}{q^{42}} .
$$

This bound built off work from his previous paper [12], where he showed that $\left\|\mathrm{e}^{n}\right\|>$ $n^{-33 n}$, where $\|x\|$ is the distance from $x$ to the nearest integer. It is still a very interesting and open question as to whether or not there is a $c>0$ such that $\left\|\mathrm{e}^{n}\right\|>c^{-n}$.

5. Concerning Diophantine equations, in [9] Mahler provided finiteness results for the number of solutions of the so-called ThueMahler equations. In particular, he showed that if $F(X, Y) \in \mathbb{Z}[X, Y]$ is an irreducible homogeneous binary form of degree at least three, $b$ is a nonzero rational integer, and $p_{1}, \ldots, p_{s}(s \geq 0)$ are distinct rational prime numbers, then the equation

$$
F(x, y)=b p_{1}^{z_{1}} \cdots p_{s}^{z_{s}},
$$

in

$$
\begin{array}{r}
x, y, z_{1}, \ldots, z_{s} \in \mathbb{Z} \text { with } \operatorname{gcd}(x, y)=1 \\
\text { and } z_{1}, \ldots, z_{s} \geq 0,
\end{array}
$$

has only finitely many solutions. The case $s=0$ was proved by Thue, corresponding to Thue equations. Mahler was the first to see the importance of extending results in Diophantine approximation to include $p$-adic valuations as well as the ordinary absolute value.
6. Mahler also made outstanding contributions to the theory of polynomials with integer coefficients. In [14], [15] he introduced what is now called the Mahler measure of a polynomial. A celebrated open question is Lehmer's problem (from an article of 1933) asking, in different words (!), whether there exists $c>1$ such that the Mahler measure of a noncyclotomic polynomial is always at least $c$.

Lehmer's Problem. Let $P(X)=a_{d} X^{d}+\cdots+$ $a_{1} X+a_{0}=a_{d}\left(X-\alpha_{1}\right) \cdots\left(X-\alpha_{d}\right)$ be a polynomial with integer coefficients. Its Mahler measure $M(P)$ is defined by

$$
M(P)=\left|a_{d}\right| \prod_{i=1}^{d} \max \left\{1,\left|\alpha_{i}\right|\right\} .
$$

Does there exist a positive real number $\varepsilon$ such that if $M(P)<1+\varepsilon$, then all the nonzero roots of $P(X)$ are roots of unity?

7. One of the most famous contributions of Mahler in geometry of numbers is his compactness theorem, established in 1946 in [11]. This is a criterion for the existence of a convergent subsequence of lattices in a sequence of lattices in the $n$-dimensional space, which may be said to have completely transformed the subject. The last words express Cassels's opinion on page 136 of his monograph [4].

\section{Conclusion}

Here we have highlighted only a few of Mahler's many mathematical contributions. We apologize if we have left out your favorite, but in this case we encourage you to find its original paper in the Archive and enjoy Mahler's own words about it; we hope that you find them in a language with which you are familiar, though this may not be the case. Indeed, like many mathematicians during Mahler's active years (1927-88), he wrote in several languages. Using the Archive as our data, we found that he published papers in his native German, as well as in English, Italian, Dutch, Russian, and even Chinese!

Mahler's interest in Chinese led him to do more than write just one paper in the language. Mahler felt so strongly that students should attempt to learn Chinese and be able to at least read mathematically in the language that while on his hiatus from Australia at Ohio State University, he suggested that Chinese be taught to graduate students in mathematics. As the story goes, he was asked who could they ever find to teach such a course? He then answered that he would do it! And indeed, Mahler gave this course. For those interested, his lecture notes, "Lectures on the 
reading of mathematics in Chinese," can be found on the Archive in the collection under the year 1972.

One can only speculate that Mahler saw the dawn of the Asian century coming. Indeed, there is a Mahler-Needham collection ${ }^{9}$ at the ANU housing Mahler's Chinese mathematics collection. Joseph Needham (1900-95) was the leading western sinologist of the past century and there was a considerable correspondence between the two concerning Volume 3 (Chinese mathematics) of Needham's 28-volume opus Science and Civilisation in China.

In addition to Mahler's formal mathematical work, we found some very interesting private writings of his. In particular, much of his correspondence with Alf van der Poorten survives. It is evident from what we have been given that Mahler and van der Poorten had a tremendous correspondence relationship, which as described a bit before, centered around mutual interests in science fiction and mathematics. These letters reveal more about the man. In a letter dated March 1, 1985, Mahler mentions his thoughts about the Riemann hypothesis and a purported proof then circulating.

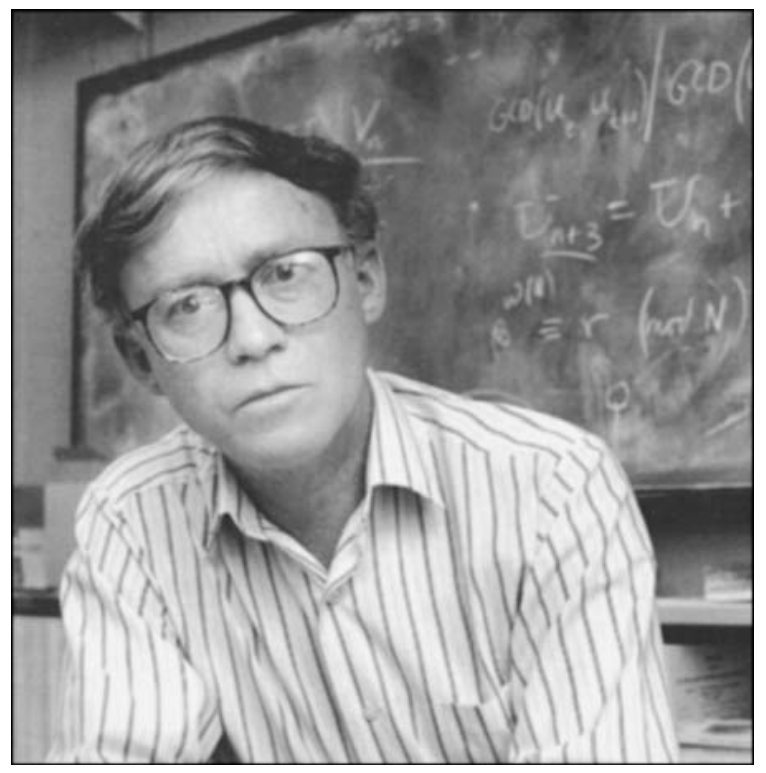

Alfred (Alf) Jacobus van der Poorten (1942-2010).

It will be a pity if the proof of Riemann's conjecture turns out to be incomplete. As you know, I am not convinced that $R C$ is true. The numerical results go only to a limit which is relatively small when we think of numbers like $10^{10^{10}}$.

-(Kurt Mahler, to Alf van der Poorten on March 1, 1985)
More so than in Mahler's papers, in his correspondence with van der Poorten, we find that Mahler was deeply interested in computational mathematics and specifically what is more commonly thought of today as experimental mathematics.

I am interested in the problem of whether there are squares of integers which, to the base $g=5$, have only digits 0 or 1 . I could not find a single example although I went quite far on my calculator. Strangely, to the base $g=7$ I obtained the one example $20^{2}=$ 1111 (7), and I am now seeing whether there are others.

- (Kurt Mahler to Alf van der Poorten on February 15, 1988)

Even in his last letter, the day before his death, Mahler was doing mathematics, lamenting that he was too old to learn to program well!

If I were ten years younger, I should also try to learn [to] handle big computers. But I have used only programmable calculators which I found very convenient. In the calculations for Squares to the base 3 I used mostly a TI 59 with printer and so could get my results. I have now also a[n] H-P 28c calculator which works to 12 places. Unfortunately the manuals that come with this machine are far too short and badly arranged. So far I have not yet been able to construct on it a program which allows [me] to express a given integer or real numbers to the base $g \geq 2$, something I could do on the TI 59.

The problem of the representation of squares to the base $g \geq 5$ seems quite hard, and I hope you have more success with it than I. It would be appropriate to consider the following more general problem.

"Let $f(x)$ be a polynomial in $x$ with integral coefficients which is positive for positive $x$. Study the integers $x$ for which the representation of $f(x)$ to the base $g \geq 3$ has only digits 0 or 1 ." Here it may be sufficient to assume that the highest coefficient of $f(x)$ is a power of $g$, and that $f$ is of the second degree. For polynomials of the first degree we settled this problem in our joint paper.

- (Kurt Mahler, to Alf van der Poorten on February 24, 1988)

Kurt Mahler remained a mathematician's mathematician until the very end.

May his theorems live forever!

-(Paul Erdős, remembering Mahler in [5])

${ }^{9}$ See anu1ib.anu.edu.au/about/co11ections/spco11. html 


\section{References}

1. JONATHAN M. BORWEIN, IGOR SHPARLINSKI, and WADIM ZUDILIN (eds.), Number theory and related fields. In memory of Alf van der Poorten. Based on the proceedings of the international number theory conference, Newcastle, Australia, March 12-16, 2012, Springer Proceedings in Mathematics \& Statistics, 43, Springer, New York, 2013 (English).

2. J. W. S. CASSELS, Obituary of Kurt Mahler, Acta Arith. 58 (1991), no. 3, 215-228. MR 1121083 (92g:01069a)

3. __ Obituary: Kurt Mahler, Bull. London Math. Soc. 24 (1992), no. 4, 381-397. MR 1165384 (93f:01016)

4. An Introduction to the Geometry of Numbers, Classics in Mathematics, Springer-Verlag, Berlin, 1997. Corrected reprint of the 1971 edition. MR 1434478 (97i:11074)

5. PAUL ERDős, Some personal and mathematical reminiscences of Kurt Mahler, Austral. Math. Soc. Gaz. 16 (1989), no. 1, 1-2. MR 992512 (90c:01068)

6. DAVID HuNT, Life and Mathematics of Alfred Jacobus van der Poorten (1942-2010), Number Theory and Related Fields (Jonathan M. Borwein, Igor Shparlinski, and Wadim Zudilin, eds.), Springer Proceedings in Mathematics \& Statistics, vol. 43, Springer, New York, 2013, pp. 1-53 (English).

7. C. G. LEKKERKERKER, In memoriam Professor Kurt Mahler, Nieuw Arch. Wisk. (4) 7 (1989), no. 1-2, 135-138. MR 1011633 (90i:01045)

8. K. MAHLER, Zur Approximation der Exponentialfunktion und des Logarithmus. I, J. Reine Angew. Math. 166 (1931), 118-136 (German).

9. __ Zur Approximation algebraischer Zahlen. I. Über den grössten Primteiler binärer Formen, Math. Ann. 107 (1933), 691-730 (German).

10. __ Arithmetische Eigenschaften einer Klasse von Dezimalbrüchen, Proc. Akad. Wet. Amsterdam 40 (1937), 421-428 (German).

11. I. Existence theorems, Proc. Roy. Soc. London. Ser. A 187 (1946), 151-187. MR 0017753 (8,195f)

12. $ـ$, On the approximation of logarithms of algebraic numbers, Philos. Trans. Roy. Soc. London. Ser. A 245 (1953), 371-398. MR 0052471 (14,624g)

13. $\_$, On the approximation of $\pi$, Nederl. Akad. Wetensch. Proc. Ser. A 56=Indagationes Math. 15 (1953), 30-42. MR $0054660(14,957 a)$

14. __ An application of Jensen's formula to polynomials, Mathematika 7 (1960), 98-100. MR 0124467 (23 \#A1779)

15. __ On some inequalities for polynomials in several variables, J. London Math. Soc. 37 (1962), 341-344. MR 0138593 (25 \#2036)

16. _ Fifty years as a mathematician, J. Number Theory 14 (1982), no. 2, 121-155. MR 655723 (84a:01051)

17. 58 (1991), no. 3, 229-237. MR 1121084 (92g:01069b)

18. _ Arithmetische Eigenschaften der Lösungen einer Klasse von Funktionalgleichungen, Math. Ann. 101 (1929), no. 1, 342-366. MR 1512537

19. B. H. NeUmANn and ALF VAN DER POORTEN, Kurt Mahler: 1903-1988, Austral. Math. Soc. Gaz. 15 (1988), no. 2, 25-27. MR 935857 (89b:01055)

20. Wolfgang M. Schmidt, T-numbers do exist, Symposia Mathematica, Vol. IV (INDAM, Rome, 1968/69),
Academic Press, London, 1970, pp. 3-26. MR 0279043 (43 \#4769)

21. __ Mahler's T-numbers, 1969 Number Theory Institute (Proc. Sympos. Pure Math., Vol. XX, State Univ. New York, Stony Brook, NY, 1969), Amer. Math. Soc., Providence, RI, 1971, pp. 275-286. MR 0344204 (49 \#8944)

22. A. J. VAN DER POORTEN, Obituary: Kurt Mahler, 19031988, J. Austral. Math. Soc. Ser. A 51 (1991), no. 3, 343-380. MR 1125440 (93a:01055)

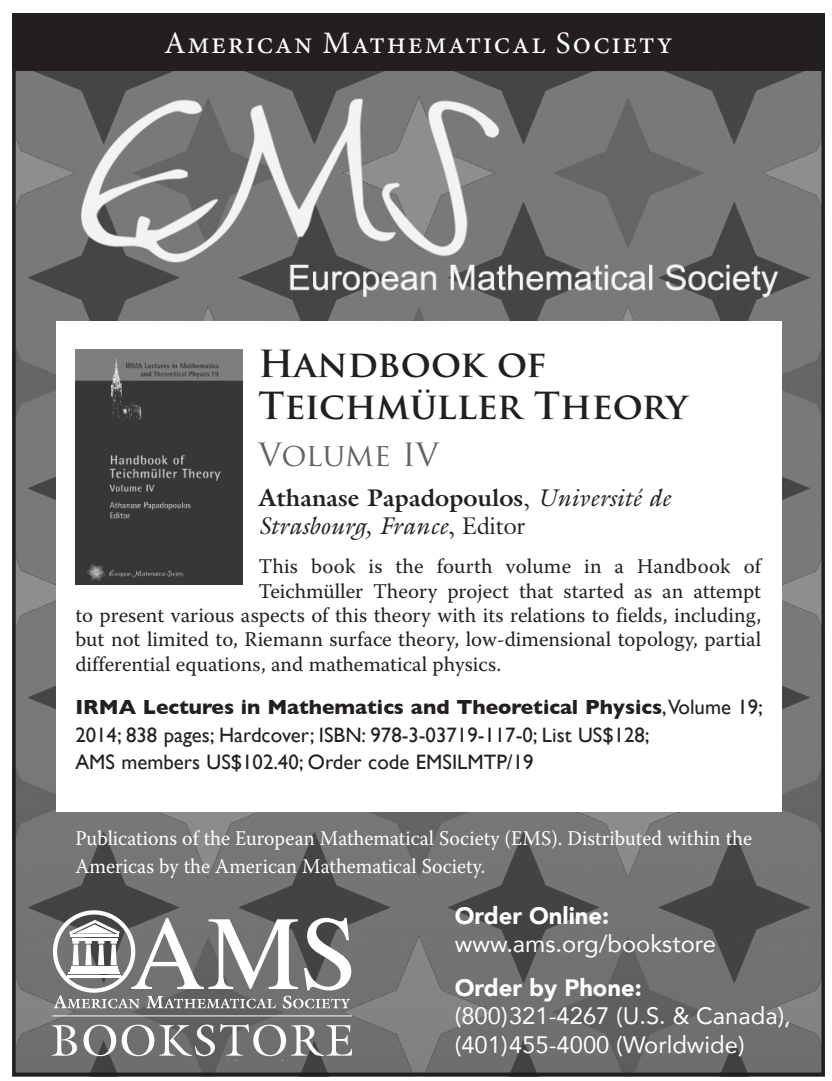

